\title{
Mise en place d'un guide d'auto-évaluation sur la maîtrise du risque radiologique
}

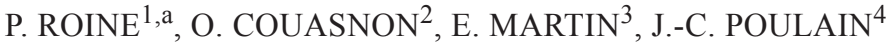

RÉSUMÉ

Le programme d'auto-évaluation basé sur la réglementation permet d'étudier celle ci, de mieux la comprendre pour faire évoluer les pratiques de l'entreprise et ses outils. La méthode est basée sur l'élaboration de processus intégrant la détermination des moyens nécessaires. Les critères décrits permettent d'évaluer l'action. La démarche s'appuie sur le référentiel MASE, associée à la norme ISO 9001. Quatre sous processus sont définis : une politique volontaire de l'entreprise, l'implication des professionnels concernés, l'association des professionnels compétents et le programme de surveillance. Neuf critères d'évaluation permettent de mesurer l'efficacité des processus, et leur amélioration continue. Parmi ces critères figurent l'organisation de la radioprotection, les ressources humaines, la formation, le suivi du personnel.

ABSTRACT Establishment of a self-assessment guide on the control of radiation risk.

The program of self assessment based on the regulation makes it possible to study it, to better include/understand it to make evolve/move the practices of the company and its tools. The method is based on the development of process integrating the determination of the means necessary. The described criteria make it possible to evaluate the action. The step is based on reference frame MASE, associated the standard ISO 9001. Four processes are defined: a voluntary policy of the company, the implication of the professionals concerned, the association of the qualified professionals and the program of monitoring. Last nine criteria of evaluation make it possible to measure the effectiveness of the processes, and their continuous improvement. Among these criteria appears the organization of protection against radiation, human resources, the training, and the follow-up of the personnel.

Keywords: Industrial radiography / self assessment / MASE / ISO 9001

\section{Pourquoi la mise en place d'une auto-évaluation?}

La radiographie industrielle met en œuvre des sources radioactives de haute activité dans des conditions de travail parfois précaires. De ce fait, elle présente un risque d'exposition non négligeable dans les conditions normales d'emploi mais aussi en situation dégradée. Pour ces raisons, cette activité est considérée à risque. Les entreprises doivent donc mettre en œuvre une politique efficace de prévention.

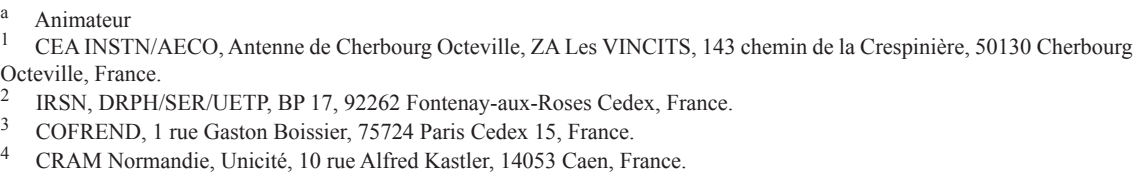


Par ailleurs, la réglementation s'est considérablement renforcée depuis la transposition de la directive européenne 96-29. Elle est, encore aujourd'hui en cours d'évolution.

Un arsenal réglementaire est désormais établi et doit être respecté par les utilisateurs et notamment, les entreprises de radiologie industrielle.

L'objectif de cette réglementation est la protection contre le risque radiologique des travailleurs, du public et de l'environnement. Sa mise en application nécessite de l'étudier, de la comprendre et éventuellement, d'adapter ses outils. Aussi, pour atteindre un niveau d'exigence suffisant, une démarche rigoureuse doit être établie. Cela implique méthode, efficacité et investissement des personnes concernées. L'auto-évaluation peut être un outil pertinent pour la satisfaction de ces trois exigences.

\section{Comment mettre en place l'auto-évaluation?}

Constat : actuellement, il est important de noter que les entreprises font des efforts pour mettre en place des procédures permettant de prendre en compte la nouvelle réglementation et tenir un référentiel documentaire en agence. On constate néanmoins que les pratiques sur le terrain ne sont pas toujours au niveau des attentes espérées.

L'application de la réglementation et une partie de la mise en place des bonnes pratiques passent par un travail en amont fait en agence mais aussi par un travail de prévention sur chantier. Il est nécessaire d'assurer un véritable encadrement des équipes qui travaillent de jour comme de nuit.

La maîtrise du risque ne peut être complète que si tous les acteurs participent activement à la mise en pratique de ce guide. Ainsi, il est important que les donneurs d'ordres s'impliquent dans cette démarche.

\subsection{Description de la méthode}

Le processus d'auto-évaluation peut être mis en œuvre d'une manière générale dans une entreprise. Il est intégré dans le processus global de qualité de l'entreprise et sa mise en place aura de ce fait un impact plus important. Afin de pouvoir être appliqué par un plus grand nombre de société, une problématique a été dégagée et une étude plus complète a été effectuée sur la maîtrise du risque radiologique.

La première partie de ce guide présente une démarche d'auto-évaluation devant permettre de maîtriser le risque radiologique. Nous proposons de la développer 
étant entendu que chaque entreprise doit se l'approprier, la compléter et l'adapter à ses besoins.

La démarche indiquée décrit l'élaboration du processus d'auto-évaluation. Elle définit ensuite des critères d'évaluation permettant d'apprécier la maîtrise globale du risque radiologique.

La deuxième partie, plus générale, est une reprise de la norme ISO 9001 (voir Tab. XV) et peut s'appliquer à toute entreprise mettant en œuvre une politique qualité. Les principaux thèmes présentés correspondent à un extrait de la partie auto-évaluation proposée dans cette norme. Pour chaque thème, des critères sont présentés. Pour chaque critère un niveau de performance minimal doit être atteint. Ce niveau a été fixé pour obtenir, a minima, la maîtrise du critère.

La politique d'amélioration continue doit permettre au cours du temps de se rapprocher, voire d'atteindre un niveau d'excellence pour certains critères.

\section{2. Élaboration d'un processus d'auto-évaluation (Tabs. I à IV)}

La politique générale de l'entreprise permet d'élaborer et de mettre en place le processus d'auto-évaluation. Ce processus se décline par la mise en œuvre d'actions qui doivent en garantir sa maîtrise. Ces actions sont résumées ainsi :

- mise en place d'une politique volontaire de l'auto-évaluation ;

- élaboration d'un programme d'activité périodique et définition des moyens nécessaires pour sa mise en œuvre ;

- création d'un comité d'évaluation et implication des professionnels concernés ;

- suivi de la mise en œuvre du programme d'activité et élaboration d'une stratégie de surveillance.

\subsection{Définition des critères d'auto-évaluation (Tabs. V à XIV)}

L'auto-évaluation repose sur plusieurs critères qui permettent d'évaluer le niveau de performance de l'entreprise de radiographie et du donneur d'ordre sur la prise en compte du risque radiologique. La méthode propose d'évaluer les thèmes suivants :

- l'organisation de la radioprotection,

- les ressources humaines liées à cette activité,

- les compétences du personnel,

- le suivi des sources et du matériel,

- le programme de contrôle radiologique,

- les mesures de prévention, l'évaluation des risques et le zonage radiologique,

- le suivi du personnel, 
- la prise en compte des situations anormales,

- la procédure chantier,

- la politique mise en place dans les entreprises utilisatrices.

\section{4. Évaluation de l'action}

Le niveau de performance de chaque critère est déterminé par l'évaluation de plusieurs sous-critères qui lui sont appliqués. Dans certains cas des indicateurs sont précisés et des objectifs sont fixés. L'entreprise pourra élaborer une grille d'évaluation afin de quantifier l'action menée par critère et sous critère. Ainsi, l'appréciation globale est rendue.

Des actions d'amélioration sont proposées afin d'augmenter le niveau de performance des sous critères et des critères. En fonction des résultats, le programme d'action élabore les priorités pour la période suivante.

Si un critère est particulièrement peu performant, des actions immédiates doivent être mises en place afin de remédier aux insuffisances relevées.

L'objectif final doit permettre d'atteindre une maîtrise optimisée du risque radiologique en toutes circonstances.

\section{Qui met en place le processus d'auto-évaluation ?}

L'évaluation des pratiques d'un établissement prendra tout son sens si des personnes extérieures à l'activité opérationnelle, bénéficiant des compétences reconnues, procèdent à l'analyse des actions mises en place. La nécessité d'associer autant que possible, des personnes extérieures au service se justifie par la difficulté d'être juge et parti. Cependant, l'efficacité sera accrue si le personnel d'encadrement concerné s'implique aussi dans la mise en place du processus d'auto-évaluation. La méthode préconise la création d'un comité de suivi et d'évaluation réunissant des personnes extérieures et une partie de l'encadrement. Le comité définit la politique d'évaluation, élabore le programme et fixe la période de sa mise en œuvre. Il prépare une grille d'auto-évaluation et analyse les résultats. Il détermine des axes de progrès et les actions à mener pour accroître la maîtrise du risque radiologique.

\section{Les référentiels existants}

Les référentiels «qualité » existants comportent généralement un chapitre sur l'auto-évaluation. De nombreuses entreprises de radiographie industrielle reposent leur politique qualité sur la démarche indiquée dans le Manuel d'Amélioration 
Sécurité des Entreprises (référentiel MASE). La démarche globale de la politique qualité dans l'entreprise peut se référer à la norme ISO 9001 dans sa dernière version. Les deux référentiels peuvent être complémentaires et dans chaque cas, le processus d'auto-évaluation dont fait l'objet ce guide peut être appliqué.

Le guide d'auto-évaluation a été conçu pour aider les utilisateurs dans la mise en place de ce processus. Chaque entreprise pourra le compléter en adaptant les critères déjà définis et les niveaux de performance en fonction de son état d'avancement. Elle adaptera, dans la mise en place du processus, les indicateurs afin de mieux apprécier, selon les utilisateurs, les critères. L'entreprise définira des priorités d'actions en respectant le principe d'une politique d'amélioration continue.

\section{TABLEAU I}

Organisation de l'auto-évaluation, sous processus 1. L'établissement initie et met en œuvre une politique coordonnée et volontaire de maîtrise du risque radiologique sur chantier, en entreprises extérieures et dans l'établissement.

The establishment initiates and implements a coordinated and voluntary control of radiation risk on site, in outside firms and in the establishment.

\begin{tabular}{ccccc}
\hline Méthodologie mise en place & Oui & $\begin{array}{c}\text { En } \\
\text { cours }\end{array}$ & Non $\quad ? \quad$ Commentaires \\
\hline
\end{tabular}

P1-1 La politique de maîtrise du risque radiologique se traduit par la définition d'un programme d'activités périodiques.

P1-2 Le programme d'activités définit l'ensemble des critères et sous critères qui garantit la maîtrise du risque radiologique. Ces critères portent sur l'organisation, les ressources humaines, l'application des 3 principes de la radioprotection, justification, optimisation et respect des limites de dose, et les mesures de prévention des risques.

P1-3 Le programme définit les moyens nécessaires pour sa mise en œuvre. Il définit notamment les moyens humains, le temps requis et les unités d'œuvre nécessaires, les moyens matériels.

P1-4 Le programme élabore un plan d'action permettant le contrôle et l'évaluation de chaque critère dans l'établissement mais aussi lors d'intervention extérieure. Pour chaque critère, son niveau de performance est identifié et des besoins particuliers sont mis en place. Le programme fixe des niveaux de performance et définit les actions à mettre en œuvre pour atteindre le niveau requis.

P1-5 Les résultats font l'objet d'un rapport qui indique l'état global de la maîtrise du risque radiologique dans l'entreprise, précise le pourcentage d'objectif atteint, le niveau de performance de chaque critère, les difficultés rencontrées ainsi que les axes d'amélioration à prendre en compte pour la période suivante. 
TABLEAU II

Organisation de l'auto-évaluation, sous processus 2. La politique de maîtrise du risque radiologique associe dans sa définition et sa mise en œuvre les professionnels concernés. The policy to control radiological risk associates in its definition and its implementation professionals involved.

\begin{tabular}{lcccc}
\hline Méthodologie mise en place & Oui & $\begin{array}{c}\text { En } \\
\text { cours }\end{array}$ & Non $\quad$ Commentaires \\
\hline
\end{tabular}

P2-1 Un comité de suivi et d'évaluation est constitué. Il est composé du chef d'établissement ou de son représentant, de la PCR, des responsables de service et du responsable HSQ ou QSE et autant que de besoin, du médecin du travail. Si possible, une personne extérieure à l'établissement et ayant des compétences reconnues pourra être associé au comité :

- il élabore la politique de maîtrise du risque radiologique et définit le programme d'activité,

- $\quad$ il met en place le programme et organise son suivi,

- $\quad$ il procède à l'évaluation de son action et identifie des axes de progrès,

- $\quad$ il met en place des actions de communication portant sur la présentation du rapport d'activité

P2-2 Les modalités de mise en œuvre du programme de maîtrise du risque radiologique permettent de garantir la coordination des actions menées par les différents professionnels. Une définition des responsabilités de chaque catégorie, connue de l'ensemble des acteurs, est élaborée.

P2-3 Le comité de suivi et d'évaluation, veille au suivi de la politique de l'établissement et à la communication des informations détenues à l'ensemble des secteurs d'activités techniques et administratives.

P2-4 Le comité de suivi et d'évaluation, est consulté pour tout projet d'aménagement des locaux, d'acquisition d'équipement ou de matériel et pour la programmation des travaux, dans le cas où ces projets ou ces choix pourraient avoir des conséquences en terme d'hygiène, de sécurité classique et radiologique.

P2-5 Le comité de suivi et d'évaluation, rédige le rapport d'activité annuel et l'adresse à la direction, aux secteurs d'activités avec lesquels il collabore et au CHSCT ou représentant du personnel le cas échéant.

TABLEAU III

Organisation de l'auto-évaluation, sous processus 3. Le programme de maîtrise du risque radiologique associe les professionnels compétents.

The program of control radiological risk associates the qualified professionals.

\begin{tabular}{llcl}
\hline Méthodologie mise en place & Oui $\begin{array}{c}\text { En } \\
\text { cours }\end{array}$ Non ? Commentaires \\
\hline
\end{tabular}

P3-1 Un responsable est identifié au sein de l'établissement et est chargé de la mise en œuvre du programme de maîtrise $\mathrm{du}$ risque radiologique.

P3-2 Le responsable a accès à toutes les données nécessaires

à la mise en œuvre du programme. Il rend compte

régulièrement de son action au comité d'évaluation. 


\section{Méthodologie mise en place}

Oui $\quad$ En

P3-3 Le programme de maîtrise du risque radiologique est intégré dans la prise en compte de l'ensemble des risques industriels auxquels l'entreprise peut être confrontée, au sein même de l'entreprise et sur les sites pour lesquels elle intervient. À ce titre, le responsable collabore avec l'ingénieur «Hygiène Sécurité » ainsi les personnes désignées par les chefs des établissements utilisatrices. Il s'assure que la coordination des travaux garantit la sécurité des intervenants.

P3-4 Le comité de suivi et d'évaluation collabore avec le médecin du travail sur toutes les questions relatives à la santé des travailleurs, à la formation à la radioprotection et l'information du personnel sur les risques liés à l'emploi des rayonnements ionisants, au suivi dosimétrique du personnel et à la mise à jour de la fiche d'exposition.

\section{TABLEAU IV \\ Organisation de l'auto-évaluation, sous processus 4. Le programme de maîtrise du risque radiologique comporte une surveillance. The program of control of the radiological risk includes monitoring.}

\begin{tabular}{lcccc}
\hline Méthodologie mise en place & Oui & $\begin{array}{c}\text { En } \\
\text { cours }\end{array}$ & Non $\quad ? \quad$ Commentaires \\
\hline P4-1 Une stratégie de
\end{tabular}

P4-1 Une stratégie de surveillance de l'activité est élaborée. Cette stratégie porte sur la mise en place de dispositif de surveillance permettant d'assurer :

- l'application du principe de justification,

- la mise en place de l'évaluation des risques,

- la mise en place de l'analyse de chaque poste de travail,

- le suivi et le classement du personnel,

- la gestion et la maîtrise de la dosimétrie individuelle et collective,

- le respect des limites de dose,

- l'application du principe d'optimisation,

- l'alerte en cas de dépassement de dose.

P4-2 Des audits sont mis en place pour évaluer :

- l'organisation,

- les procédures et leur mise en œuvre,

- les compétences du personnel et leur connaissance se rapportant au métier, aux règles de radioprotection, aux consignes de sécurité générales et particulières à un site donné,

- le déroulement des chantiers, chez les grands donneurs d'ordres, chez les tuyauteurs et chaudronniers, dans l'établissement.

P4-3 Des audits sont mis en place afin de s'assurer que les consignes de sécurité sont correctement appliquées sur chantier et le cas échéant, dans l'établissement. Cela oblige la mise en place d'actions de contrôle.

P4-4 Le comité de suivi pilote cette surveillance et analyse les résultats des actions qui sont menées. Il fixe le nombre et le thème des audits ainsi que le calendrier des actions de contrôles. Les conclusions de cette surveillance sont 


\begin{tabular}{|c|c|c|c|c|c|}
\hline Méthodologie mise en place & Oui & $\begin{array}{c}\text { En } \\
\text { cours }\end{array}$ & Non & $?$ & Commentaires \\
\hline $\begin{array}{l}\text { communiquées aux secteurs d'activité concernés et à la } \\
\text { direction. Elles permettent de dresser un bilan de l'état } \\
\text { de la maîtrise du risque radiologique et de faire des } \\
\text { recommandations pour l'amélioration de sa prise en compte. } \\
\text { Ces conclusions sont utilisées pour déterminer des axes de } \\
\text { progrès par thématique, pour élaborer le programme de suivi } \\
\text { et pour conduire des actions de prévention. }\end{array}$ & & & & & \\
\hline
\end{tabular}

TABLEAU V

Critères d'auto-évaluation, critère 1 : évaluation de l'organisation de la radioprotection. Le programme de maîtrise du risque radiologique repose sur une bonne organisation de la radioprotection.

Assessment of management of radioprotection: the program of control of the radiological risk rests on a good organization of protection against radiation.

\begin{tabular}{llcll}
\hline Méthodologie mise en place & Oui & $\begin{array}{c}\text { En } \\
\text { cours }\end{array}$ & Non $\quad$ Commentaires \\
\hline
\end{tabular}

C1-1 L'organisation de la radioprotection et la coordination des actions sont évaluées :

- un organigramme fonctionnel définit les rôles et les responsabilités de chaque personne intervenant autour des sources missions de chaque personne ;

- les fiches de fonction de chaque métier sont formalisées ;

- les fiches de poste sont effectuées pour tout le personnel et font apparaître les risques inhérents à la profession.

C1-2 Le service compétent en radioprotection est évalué. Les missions de chaque personne compétente en radioprotection sont précisées et formalisées.

Des objectifs sur la radioprotection leur sont fixés. Ils

concernent les actions :

- $\quad$ sur la mise en place du programme de contrôle ;

- $\quad$ sur l'évaluation des risques, le zonage et le calcul des zones d'opération sur chantier ;

- $\quad$ sur la formation à la radioprotection ;

- $\quad$ sur les études de poste ;

- sur le suivi et l'encadrement du personnel, la dosimétrie active, les évaluations prévisionnelles de dose ;

- $\quad$ sur le recensement des situations pouvant entraîner un dépassement de dose ;

- sur la participation à la mise en place d'un plan d'urgence interne.

Les actions communes avec le médecin du travail ainsi que le CHSCT ou délégué du personnel sont définies

C1-3 La préparation et l'organisation des travaux utilisant les rayonnements sont évaluées. Les affaires et les chantiers sont organisés. Les conditions de chantiers sont évaluées. Des objectifs en terme de moyens, de personnel, de prévisionnel de dose sont fixés et suivis. 


\begin{tabular}{|c|c|c|c|c|c|}
\hline Méthodologie mise en place & Oui & $\begin{array}{c}\text { En } \\
\text { cours }\end{array}$ & Non & $?$ & Commentaires \\
\hline $\begin{array}{l}\text { C1-4 Le plan d'urgence interne est mis en place et } \\
\text { évalué. Les situations exceptionnelles sont recensées. Les } \\
\text { conséquences sont analysées. Des fiches réflexes sont mises } \\
\text { à disposition des opérateurs et leur mise en œuvre fait l'objet } \\
\text { d'exercice. }\end{array}$ & & & & & \\
\hline
\end{tabular}

C1-5 En cas d'incident ou d'accident, les causes sont analysées et donnent lieu à un retour d'expérience. Le REX est évalué.

\section{Indicateurs}

Nombre d'actions de prévention effectué par chaque PCR.

Nombre d'actions de formation.

Nombre d'unités d'œuvre effectué pour la mise en place de la radioprotection.

\section{TABLEAU VI}

Critères d'auto-évaluation, critère 2 : évaluation des ressources humaines. Le programme de maîtrise du risque radiologique repose sur une politique des ressources humaines cohérentes et sur la compétence du personnel.

Assessment of human resources: the program of control radiological risk rests on a policy of coherent human resources and on the competence of the personnel.

\begin{tabular}{llccc}
\hline Méthodologie mise en place & Oui & $\begin{array}{c}\text { En } \\
\text { cours }\end{array}$ & Non $\quad$ Commentaires \\
\hline
\end{tabular}

C2-1 Le programme évalue les besoins en ressources

humaines :

Il tient compte :

- de la charge de travail ;

- des délais et durées d'intervention :

- des distances d'intervention des chantiers.

C2-2 Le programme évalue les aptitudes du personnel :

- le personnel utilisant les sources de rayonnements dispose de son aptitude médicale à jour ;

- la catégorie du personnel est fonction de son poste de travail et de la dose efficace reçue sur les 12 derniers mois.

C2-3 Le programme évalue la prise en charge des nouveaux arrivants :

- leur prise en charge est décrite dans une procédure ;

- leurs formations et leur aptitude médicale sont programmées ;

- leurs compétences et ses capacités d'adaptation sont suivies et évaluées.

\footnotetext{
Indicateurs

- Temps effectif de travail des radiologues.

- Nombre d'écarts relevés sur les dépassements des périodicités et sur le suivi de l'aptitude médical.

- Nombre d'écart entre le classement et la dose reçue annuellement par travailleur.

- Temps nécessaire pour délivrer l'aptitude médicale et la formation initiale d'un nouvel arrivant.
} 
TABLEAU VII

Critères d'auto-évaluation, critère 3 : évaluation du plan de formation.

Le programme de maîtrise des risques radiologiques s'assure que le plan de formation garantit les compétences nécessaires des opérateurs.

Assessment of training program: the program of control of the radiological risks makes sure that the training program guarantees necessary the skills of operators.

\begin{tabular}{llcll}
\hline Méthodologie mise en place & Oui & $\begin{array}{c}\text { En } \\
\text { cours }\end{array}$ & Non $\quad$ Commentaires \\
\hline
\end{tabular}

C3-1 Le programme de maîtrise du risque radiologique permet de s'assurer que le plan de formation mis en place dans l'entreprise correspond au besoin de l'entreprise et aux exigences réglementaires :

Ce plan de formation est dispensé à tout professionnel, intérimaire, CDD ou CDI lors de son arrivée. Il correspond, a minima, aux exigences réglementaires à savoir :

- formation à la radioprotection pour toutes personnes exposées aux rayonnements ionisants ;

- formation CAMARI pour les personnes devant manipuler les appareils de radiographie industrielle ;

- formation transport classe 7 pour chaque chauffeur amené à transporter des sources radioactives ;

- formation sécurité dans les entreprises clientes pour tous les intervenants chantiers.

Ce plan fait partie d'un processus de management des connaissances et de capitalisation d'expérience qui doit être défini et déployé.

C3-2 Le programme de maîtrises des risques radiologiques s'assure que les actions de formation continue en radioprotection concernent l'ensemble des professionnels concernés et qu'elles sont provoquées lors des renouvellements périodiques obligatoires, des évolutions de la réglementation, la diffusion de nouvelles procédures et la modification du risque pour un poste de travail considéré (intervention sur un site nouveau pouvant présenter des risques particuliers).

C3-3 Le programme évalue les compétences du personnel

- $\quad$ il permet de s'assurer que les radiologues ont suivi les formations obligatoires et leurs renouvellements ;

- il s'assure que les formations complémentaires sont mises en place et suivies ;

- $\quad$ il contient des actions permettant d'évaluer les connaissances du personnel sur la radioprotection, les procédures en place, les consignes de sécurité, les mesures d'urgence et les fiches réflexes en cas de situation dégradée et d'urgence radiologique.

\footnotetext{
Indicateurs

- Nombre d'écarts relevés sur les dépassements des périodicités de formation obligatoire.

- Nombre d'écarts relevés sur les dépassements des périodicités indiquées dans le plan de formation.

- Résultats de test d'évaluation des connaissances des travailleurs.
} 


\section{TABLEAU VIII}

Critères d'auto-évaluation, critère 4 : évaluation du suivi du personnel.

Le programme de maîtrise du risque radiologique s'assure que le suivi du personnel travaillant sous rayonnements est rigoureux.

Assessment of follow-up of personnel: the program of control of the radiological risk makes sure that the follow-up of the personnel working under radiations is rigorous.

\begin{tabular}{lllll}
\hline Méthodologie mise en place & Oui & $\begin{array}{c}\text { En } \\
\text { cours }\end{array}$ & Non & Commentaires \\
\hline
\end{tabular}

C4-1 Une procédure sur le suivi des travailleurs existe, et son application fait l'objet d'une évaluation.

C4-2 Les analyses de chaque poste de travail sont effectuées, régulièrement remises à jour et évaluées :

- les fiches d'exposition existent et sont transmises au médecin du travail ;

- la catégorie de chaque travailleur est déterminée ;

le suivi dosimétrique des travailleurs est rigoureux et les résultats sont analysés régulièrement afin de prévenir des dépassements des limites réglementaires ;

- le suivi dosimétrique se fait en collaboration du médecin du travail.

C4-3 Le programme de maîtrise du risque radiologique évalue le principe d'optimisation :

- les résultats de la dosimétrie opérationnelle sont suivis et analysés et le principe d'optimisation est appliqué ;

- l'évaluation prévisionnelle de doses est effectuée et fait l'objet d'un suivi pour chaque chantier.

\section{Indicateurs}

- Nombre de postes de travail n'ayant pas fait l'objet d'une analyse.

- Nombre de postes de travail requérant un classement en catégorie $\mathrm{A}$ et en catégorie $\mathrm{B}$.

Nombre d'écarts observés sur les fiches d'exposition.

Objectif de la dose collective par affaire et de la dose annuelle.

- Objectif de la dose individuelle par poste de travail.

- Nombre d'évaluations prévisionnelles non effectuées.

- Suivi des écarts entre le prévisionnel de dose et le réalisé.

\section{TABLEAU IX}

Critères d'auto-évaluation, critère 5 : évaluation du suivi des sources et du matériel. Le risque radiologique lié à l'utilisation des dispositifs émetteurs de rayonnements et à son environnement est maîtrisé.

Assessment of the follow-up of radiation sources and of materiel: the radiation risk associated with the use of transmitter's devices radiation and on its environment is controlled.

$\begin{array}{llc}\text { Méthodologie mise en place } & \text { Oui } & \begin{array}{c}\text { En } \\ \text { cours }\end{array}\end{array}$ Non ? Commentaires

C5-1 Un système d'assurance de la qualité est mis en place pour le suivi du matériel et des sources de rayonnements et fait l'objet d'une évaluation lors d'audits programmés. 


\begin{tabular}{llcll}
\hline \multicolumn{1}{|c}{ Méthodologie mise en place } & Oui & $\begin{array}{c}\text { En } \\
\text { cours }\end{array}$ & Non $\quad$ Commentaires \\
\hline $\begin{array}{l}\text { C5-2 Les autorisations de détention et d'utilisation des } \\
\text { sources de rayonnements sont disponibles et à jour. Elles font } \\
\text { l'objet d'un suivi rigoureux qui est évalué. }\end{array}$ & & & \\
\end{tabular}

C5-3 Le principe de justification est évalué :

- lors de chaque changement de source ;

- lors de la préparation des chantiers ;

- lors du renouvellement d'autorisation.

C5-4 Des procédures sont écrites, validées et mises en place par du personnel formé. Leur respect et utilité sont évalués à périodicité définie. Ces évaluations donnent lieu à des révisions régulières.

Les procédures concernent :

- l'entretien de l'ensemble des locaux en fonction des zones mises en place ;

- le suivi des sources radioactives (mise en place et tenu d'un registre) ;

- l'échange et/ou la reprise des sources périmées ;

- l'entretien des projecteurs et accessoires (sur site ou en entreprises spécialisées).

C5-5 La gestion des incidents se produisant sur le matériel, l'application des consignes mises en place et le retour d'expérience qui en est fait, sont évalués.

C5-6 La gestion des incidents de sources radioactives (chantiers, transport et établissement), l'application des consignes mises en place, les actions menées par les opérateurs et le retour d'expérience qui en est fait, sont évalués.

C5-7 En cas d'anomalie de source radioactive, une procédure est écrite et précise a minima qu'un compte rendu est établi et que les causes et les circonstances sont identifiées, que les conséquences et les mesures pour remédier au problème sont mises en place. Cette procédure fait l'objet d'une évaluation.

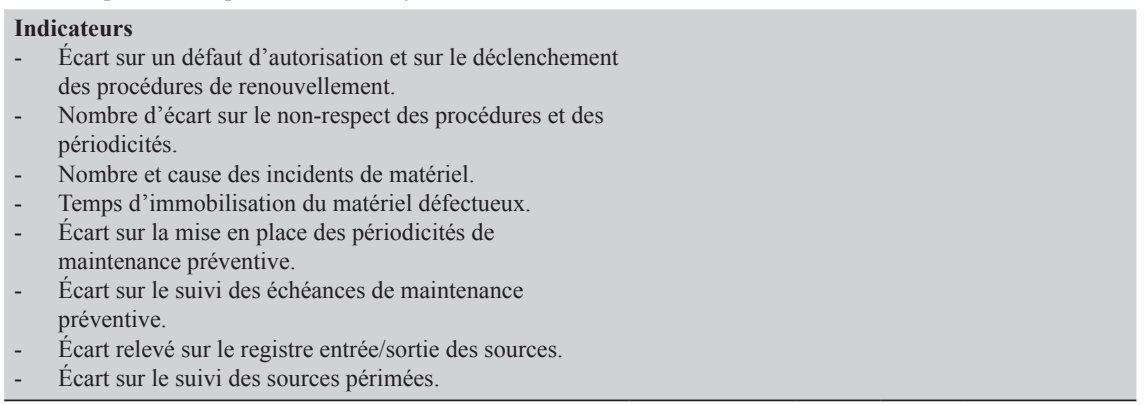


TABLEAU $X$

Critères d'auto-évaluation, critère 6 : évaluation du programme de contrôle radiologique. Le programme de maîtrise du risque radiologique s'assure que le programme est en place et que ses objectifs sont atteints.

Assessment of radiation monitoring: the program to control the radiological risk ensures that the program is in place and that its objectives are achieved.

\begin{tabular}{lcccc}
\hline Méthodologie mise en place & Oui & $\begin{array}{c}\text { En } \\
\text { cours }\end{array}$ & Non $\quad ? \quad$ Commentaires \\
\hline
\end{tabular}

C6-1 Le programme de contrôle existe et est évalué :

- les contrôles internes et externes existent et sont évalués ;

- l'ensemble des contrôles prescrit par la réglementation est mis en place ;

- leurs périodicités sont définies et justifiées ;

- les contrôles sont effectués régulièrement sur chantier ;

- les contrôles sont effectués systématiquement lors du rechargement des projecteurs ;

- les sources de hautes activités font l'objet de contrôles adaptés ;

- les appareils de mesure et plus particulièrement les dosimètres opérationnels sont inclus dans le programme de contrôle ;

- les contrôles sont repris systématiquement dans le rapport qui est laissé à la disposition des responsables d'activités.

C6-2 Le programme de maîtrise du risque radiologique s'assure que du personnel compétent effectue ces contrôles :

- la personne compétente en radioprotection effectue ces contrôles ;

- en cas d'absence, elle désigne et forme une personne pour la remplacer ;

- chaque contrôle fait l'objet d'une procédure permettant de garantir que les mesures sont reproductibles et que toutes les sécurités sont contrôlées.

C6-3 Le programme évalue la prise en charge des écarts et des non-conformités :

- chaque compte rendu issu d'un contrôle interne ou externe, fait apparaître les écarts à la réglementation, les écarts aux procédures internes et les non-conformités des installations et des projecteurs ;

- lorsqu'ils sont relevés, ils font l'objet d'un suivi jusqu'à leur résolution ;

- ces écarts et non-conformité sont tracés et les actions permettant d'y remédier font l'objet d'un suivi ;

- les moyens pour remédier aux écarts sont évalués et donnent lieu le cas échéant à un retour d'expérience.

Indicateurs

- Nombre d'écarts relevé par les contrôles internes. Nombre d'écarts relevé par les contrôles externes. Respect des périodicités de contrôle.

Nombre d'écarts sur l'application du programme de contrôle.

- Suivi du temps nécessaire à la remise en conformité des écarts. 
TABLEAU XI

Critères d'auto-évaluation, critère 7 : évaluation des risques. Le programme de maîtrise du risque radiologique évalue les actions de prévention.

Risk assessment: the program to control the radiological risk evaluates preventive measures.

\begin{tabular}{lccc} 
Méthodologie mise en place & Oui & $\begin{array}{c}\text { En } \\
\text { cours }\end{array}$ & Non ? Commentaires \\
\hline
\end{tabular}

C7-1 Le programme de maîtrise du risque radiologique s'assure que des protocoles visant à maîtriser le risque radiologique sont en place :

- les conditions d'emploi de chaque source sont recensées ;

- l'évaluation des risques autour de chaque source de rayonnements pour chaque condition d'emploi est effectuée ;

- $\quad$ les consignes de sécurité sont élaborées et évaluées ;

- les procédures d'utilisation existent et sont connues par les opérateurs et évaluées ;

- les procédures et consignes en cas d'incident et d'accident ainsi que les mesures d'urgence sont disponibles et à disposition des utilisateurs. Elles font l'objet d'évaluation régulière.

C7-2 Le programme de maîtrise du risque radiologique apprécie l'évaluation des risques mise en place autour des sources de rayonnements :

- le zonage radiologique est défini et mis en place. La cohérence vis-à-vis du risque radiologique et des résultats de mesure est évaluée ;

- la procédure de mise en place des zones d'opérations existe et pour chaque intervention sur chantier la zone d'opération est définie et réglementaire.

C7-3 L'entreprise cliente ou utilisatrice est associée aux actions de prévention du risque radiologique la concernant. Le comité de suivi du programme de maîtrise du risque radiologique évalue l'élaboration et la mise en œuvre des plans de prévention ou des plans particuliers de sécurité et de protection de la santé.

\footnotetext{
Indicateurs

- Nombre d'écarts relevé entre les résultats des contrôles et

le zonage effectif, dans l'établissement et sur chantier.

- Sources utilisées en dehors de ses conditions définies.

- Écarts relevés sur l'application des consignes sécurités, des consignes en cas de situation dégradée.

- $\quad$ Nombre de plan de prévention pas adapté ou absent.
} 
TABLEAU XII

Critères d'auto-évaluation, critère 8 : évaluation de la prise en compte des situations d'urgence et des actions nécessaires permettant de gérer ces situations. Un programme de prévention et de prise en charge des surexpositions touchant les professionnels, établi en collaboration avec la médecine du travail, est élaboré.

Assessment taking into accounts emergency situations and actions necessary to manage these situations: a program of prevention and care of overexposure related professionals, in collaboration with occupational medicine is developed.

\begin{tabular}{llc}
\hline Méthodologie mise en place & Oui & $\begin{array}{c}\text { En } \\
\text { cours }\end{array}$
\end{tabular} Non ? Commentaires

C8-1 Le plan d'urgence interne (PUI) existe et fait l'objet d'une évaluation :

- les causes possibles d'incidents ou d'accidents sont recensées et identifiées ;

- des actions sont menées pour prévenir les accidents pouvant entraîner une exposition radiologique non maîtrisée.

C8-2 Un dispositif de notification, de recueil, d'analyse de ces accidents et de prise en charge des personnes concernées est mis en place.

C8-3 L'ensemble des professionnels est formé et dispose des consignes et fiches réflexes afin d'intervenir dans les

meilleurs délais et conditions en cas d'incident ou d'accident pouvant conduire à une exposition non maîtrisée.

\section{Indicateurs}

- Nombre d'incident de radioprotection selon l'échelle expérimentale de l'ASN.

- Nombre de dépassements de dose par rapport aux objectifs fixés.

- Nombre de presque accident relevé.

- Nombre d'actions de prévention menées et résultat de ces actions.

- Évaluation sur la mise en pratique des fiches réflexes lors d'entrainements. 
TABLEAU XIII

Critères d'auto-évaluation, critère 9 : organisation des chantiers. Le programme de maîtrise du risque radiologique évalue régulièrement la radioprotection sur les chantiers extérieurs. Organization yards: the program of risk management regularly assesses radiological radiation on external sites.

\begin{tabular}{lcc}
\hline Méthodologie mise en place & Oui & $\begin{array}{c}\text { En } \\
\text { cours }\end{array}$
\end{tabular} Non ? Commentaires

C9-1 Une procédure sur l'organisation des chantiers existe et est évaluée. Elle concerne :

- la préparation du chantier ;

- les conditions d'intervention spécifique à chaque site sur

lequel l'entreprise intervient ;

- l'utilisation des sources ;

- la manipulation, l'entreposage, l'utilisation des projecteurs ;

- le repli de chantier ;

C9-2 Pour chaque chantier, un chef de chantier est désigné. Ses attributions et ses limites de compétence sont définies. Le programme de maîtrise du risque radiologique évalue régulièrement son aptitude à encadrer un chantier.

C9-3 Les délais d'intervention font l'objet d'une évaluation. En cas de délais réduits, des mesures compensatoires sont mises en place et obligent à faire appel à des radiologues disponibles, confirmés et disposant des qualifications nécessaires pour l'intervention sur ce site.

C9-4 Pour chaque chantier, un lieu d'entreposage garantissant la sécurité et la sûreté des sources est identifié.

C9-5 Un système permet de remonter les anomalies et les dysfonctionnements relevés lors du déroulement du chantier. Lors de l'application d'un droit de retrait, les motivations de son application font l'objet d'une analyse tracée.

\footnotetext{
C9-6 Chaque chantier donne lieu à l'élaboration d'un plan de prévention ou d'un plan particulier de sécurité et de protection de la santé adapté à l'intervention. Ce plan est adapté à chaque intervention.

\section{Indicateurs}

- Résultat de l'évaluation du chef de chantier.

- Écart sur les conditions d'entreposage et nombre de cas où aucun entreposage n'a été trouvé.

- Nombre de chantiers programmés dans des délais réduits.

- Nombre de droit de retrait mis en application.

- Écart sur les critères définissant les paramètres d'intervention (plan de tir, plage horaire, coactivité...).

- Mise en application des critères d'entreposage des sources radioactives sur chantier.

- Suivi du retour d'expérience.
} 


\begin{abstract}
TABLEAU XIV
Critères d'auto-évaluation, critère 10 : évaluation des donneurs d'ordres. La maîtrise du risque radiologique s'intègre dans la politique de maîtrise globale des risques de l'entreprise utilisatrice (exploitant du site).

Assessment of the clients: the control of the radiological risk is integrated in the policy of total control of the risks of the company user (operator of the site).
\end{abstract}

\begin{tabular}{|c|c|c|c|c|c|}
\hline Méthodologie mise en place & Oui & $\begin{array}{c}\text { En } \\
\text { cours }\end{array}$ & Non & $?$ & Commentaires \\
\hline
\end{tabular}

C10-1 Le donneur d'ordres est parti prenante dans les actions permettant de maîtriser le risque radiologique sur son site. Il coordonne les actions de prévention avec le comité de suivi et d'évaluation. Il désigne une personne compétente en radioprotection afin de garantir la coordination des actions.

C10-2 Le donneur d'ordres se sert du retour d'expérience en vu d'une amélioration continue pour la maîtrise du risque radiologique.

C10-3 Le donneur d'ordres recense les situations incidentelles ou accidentelles et met en place les procédures d'urgence en tenant compte de la multiplicité des risques.

C10-4 Le donneur d'ordres fixe des critères portant sur la maîtrise du risque radiologique et permettant le choix des entreprises de radiographie industrielle

C10-5 Une procédure portant sur les travaux de radiologie industrielle existe et fait l'objet d'une évaluation. Elle concerne :

- l'évaluation de l'application du principe de justification ;

- l'organisation et l'autorisation d'un chantier ;

- la coordination générale des travaux de radiographie industrielle ;

- le suivi et le déroulement de chaque chantier.

C10-6 Le donneur d'ordres évalue la compétence de son personnel. En outre, il s'assure que les équipes d'intervention disposent de la qualification et des moyens nécessaires en cas d'urgence radiologique.

C10-7 Le donneur d'ordres met en place une évaluation des actions permettant la prévention du risque radiologique. Il évalue :

- l'élaboration du cahier des charges ;

- l'élaboration du plan de prévention;

- la fourniture et la mise en place des moyens de protection collective voire individuelle pour des risques particuliers au site utilisateur ;

- les actions de prévention permettant la sensibilisation à la radioprotection ;

- la gestion de la co-activité ;

- le déroulement de chaque intervention ;

- la compatibilité du délai de commande et du délai d'intervention en fonction de la prestation à réaliser.

Pour les grands arrêts, il évalue les procédures et les moyens pour assurer la coordination des entreprises.

C10-8 Le donneur d'ordres élabore et transmet toutes les procédures de sécurité applicables sur le site. Il en évalue l'application par l'entreprise de radiographie et lui transmet régulièrement les résultats afin qu'ils soient pris en compte dans la politique d'amélioration continue. 
TABLEAU XV

Auto-évaluation de l'entreprise, partie générale.

Self-assessment of the company, general part.

\begin{tabular}{|c|c|}
\hline Critère & Niveau de performance minimal requis \\
\hline \multicolumn{2}{|l|}{ A- Responsabilité de la direction } \\
\hline Analyse initiale et écoute & Un processus d'analyse initiale est établi et systématique \\
\hline Identification des exigences légales & Une veille réglementaire est organisée au sein de l'entreprise \\
\hline Politique et objectifs & $\begin{array}{l}\text { L'engagement de la direction s'appuie sur une politique affirmée } \\
\text { déployée à chaque niveau approprié }\end{array}$ \\
\hline Planification & $\begin{array}{l}\text { L'entreprise identifie et planifie les actions et ressources nécessaires } \\
\text { pour atteindre les objectifs }\end{array}$ \\
\hline Approche processus & Les processus sont identifiés, décrits, analysés et pilotés \\
\hline Responsabilités et autorité & $\begin{array}{l}\text { Les rôles, responsabilités et autorité de tout acteur sont définies et } \\
\text { communiquées }\end{array}$ \\
\hline Communication interne & $\begin{array}{l}\text { Le système de management intègre un dispositif de communication } \\
\text { interne et externe }\end{array}$ \\
\hline Revue de direction & Des revues de direction sont planifiées et impliquent des décisions \\
\hline \multicolumn{2}{|l|}{ B- Management des ressources } \\
\hline $\begin{array}{l}\text { Management des compétences } \\
\text { de la formation }\end{array}$ & $\begin{array}{l}\text { Les ressources humaines sont centrées autour de la définition des } \\
\text { responsabilités et de la formation } \\
\text { Favorise une responsabilisation du personnel }\end{array}$ \\
\hline Management de l'information & Les informations essentielles sont partagées \\
\hline Management des ressources matérielles & $\begin{array}{l}\text { L'adéquation des ressources matérielles aux objectifs des processus est } \\
\text { recherchée. }\end{array}$ \\
\hline Environnement de travail & $\begin{array}{l}\text { L'environnement du travail garantit la conformité du produit et la } \\
\text { protection du personnel }\end{array}$ \\
\hline
\end{tabular}

\section{C- Réalisation de la prestation}

\begin{tabular}{|ll}
\hline Planification opérationnelle & $\begin{array}{l}\text { La planification permet de répondre aux exigences des clients, de la } \\
\text { réglementation et de l'entreprise }\end{array}$ \\
\hline Processus relatifs aux clients & Le processus commercial vise la satisfaction du client \\
\hline Identification et traçabilité & $\begin{array}{l}\text { La traçabilité concerne les produits ciblés et permet de retrouver les } \\
\text { conditions de réalisation du produit }\end{array}$
\end{tabular}

\section{D- Mesure, analyse, amélioration}

\begin{tabular}{ll} 
Contrôle et surveillance & $\begin{array}{l}\text { Les contrôles et surveillance ont pour objet l'optimisation de l'ensemble } \\
\text { des processus }\end{array}$ \\
\hline Performance du système & $\begin{array}{l}\text { La performance de l'entreprise est efficace } \\
\text { Amélioration continue }\end{array}$ \\
$\begin{array}{l}\text { Le déploiement des actions d'amélioration est général et permanent au } \\
\text { sein de l'entreprise. L'amélioration des performances des processus est } \\
\text { recherchée }\end{array}$
\end{tabular}

Prévention des situations d'urgence et de crise 